\title{
Jet-medium interaction and the Gubser flow
}

\author{
Li Yan*, Sangyong Jeon, and Charles Gale \\ Department of Physics, McGill University, 3600 Rue University, Montréal, QC, H3A 2T8, \\ Canada \\ E-mail: li.yan@physics.mcgill.ca
}

\begin{abstract}
We study the effects of expansion and viscous corrections on the hydrodynamical medium response to a high energy jet parton. More specifically, using a semi-analytical Gubser solution to relativistic fluid dynamics, modifications to the formed Mach cone, diffusive wake, and the momentum flow of the medium response along and perpendicular to the jet particle, are analyzed mode-by-mode. This should provide intuition and guidance for analyses of the experimentally measured jet sub-structure in heavy-ion collisions.
\end{abstract}

Critical Point and Onset of Deconfinement - CPOD2017

7-11 August, 2017

The Wang Center, Stony Brook University, Stony Brook, NY

${ }^{*}$ Speaker. 


\section{Introduction}

One of the interesting problems investigated in heavy-ion experiments carried out at Relativistic Heavy-Ion Collider (RHIC) and the Large Hadron Collider (LHC) is that of jet quenching $[1,2]$, which occurs when an energetic parton (quark or gluon) goes through dense and hot quark-gluon plasma (QGP). Analyses of jet quenching are expected to reveal fundamental properties of the strong interaction, like how an energetic parton deposits energy into the medium. In that context, the parameter $\hat{q}$ was proposed and extracted to characterize the averaged transverse momentum broadening of a jet parton propagating in the medium [3]. Recently, experiments at the LHC energies have reached an unprecedented level of details, in what concerns the analyses of jet sub-structure [4]. Those measurements and analyses extend to large jet cone radii [5], where the dominant contributions come from the jet-medium interaction. This therefore presents jet-medium interactions as a class of alternative probe of information related to the dissipative properties of the medium, such as the specific shear viscosity $\eta / s$.

A consistent theoretical description of the jet-medium interaction is challenging, but several analyses have nevertheless been performed $[6,7,8,9,10,11,12,13,14,15]$. Part of the complication owes to the fact that the jet-medium interaction involves simultaneously a hard energy scale from the jet parton, and a soft energy scale from the evolving background medium. The dynamics of the jet parton normally can be captured by perturbative QCD calculations. The evolution of the background medium, on the other hand, requires effective modelings such as viscous hydrodynamics. It is possible to take into account the effect of jet-medium interaction in the framework of hydrodynamics, as has been applied in many theoretical analyses. However, one has to be aware that the evolution of high energy modes associated with the jet parton may be beyond the validity of hydrodynamics. The key is to incorporate properly a high energy mode in hydrodynamics: a theory in which the dominant modes are long-wavelength (low energy) excitations.

In these proceedings, we present a formalism developed in Ref. [16], in which the perturbations induced by the energy deposition from an energetic parton are decomposed into modes. Then, the evolution of these modes can be studied in conjunction with an analytically solved evolution of a conformal medium, the Gubser flow. While long-wavelength modes are safely compatible with hydrodynamics, short-wavelength modes are damped as a direct consequence of viscous suppression. This is consistent with the expectation that modes of longer wavelength are more easily thermalized and absorbed in the background medium system [17]. The jet-medium interaction perturbs the hydrodynamic fields and leads leads to a conical structure. The effects of the jet-medium interaction are then examined in the generated particle spectrum, after summing over modes.

The basic aspects of the theoretical framework are discussed in Section 2, with the equation of motion describing the jet-medium interaction introduced and solved with respect to the Gubser flow. The cone induced from the jet-medium interaction is described in Section 3.

\section{Methodology}

We discuss the jet propagation through a medium in terms of the fluid dynamics, and we apply an analytically solvable model of the Gubser flow. For simplicity, in the calculations we impose 
Bjorken boost invariance, so that the background medium, as well as the jet parton, are realized as boost invariant. This is an idealization, but we shall focus on the features we expect to be robust.

\subsection{The jet-medium interaction}

In the framework of hydrodynamics, the system evolution is characterized by a set of equations that reflect conservation laws. >From the conservation of energy-momentum, one has

$$
\partial_{\mu} T^{\mu v}=0
$$

where the energy-momentum tensor $T^{\mu v}$ is a function of hydrodynamic fields: Energy density $e$, pressure $\mathscr{P}$ and flow velocity $u^{\mu}$. The system consists of the background fluid, the jet parton, and their interaction. We thus expect the energy-momentum tensor to be

$$
T^{\mu v}=T_{\text {hydro }}^{\mu v}+T_{\text {jet }}^{\mu v}+\delta T^{\mu v}
$$

where the three terms in the right hand side corresponds to the energy-momentum tensor of the background fluid, the jet parton, and the jet-medium interaction, respectively. In this work we shall treat the contributions from the jet parton to the medium as a perturbation, when comparing to the evolution of the background medium. Therefore, in linearized hydrodynamics the conservation equation Eq. (2.1) is separated as

$$
\begin{aligned}
\partial_{\mu} T_{\text {hydro }}^{\mu v} & =0, \\
\partial_{\mu}\left(T_{\text {jet }}^{\mu v}+\delta T^{\mu v}\right) & =0,
\end{aligned}
$$

where the first equation is the ordinary hydrodynamic equation of motion describing the evolution of the background fluid, and the second one focuses on the effect of jet-medium interaction. Note that at leading order the jet-medium interaction does not affect the evolution of the background fluid, so that two equations in Eq. (2.3) are decoupled. This procedure can be generalized to higher orders iteratively. The formulation involves the nonlinear couplings of the perturbations at second order, and is shown in Appendix A.

The evolution of the jet parton itself can be rewritten effectively as a source term, $J^{v}=$ $-\partial_{\mu} T_{\text {jet }}^{\mu v}$. Accordingly, for the jet-medium interaction one has

$$
\partial_{\mu} \delta T^{\mu v}=J^{v}
$$

Eq. (2.4) is the central equation we solve in this work to describe the effects of jet-medium interaction. Taking $\delta T^{\mu v}$ the linearized perturbations induced by the jet source, Eq. (2.4) can be expressed explicitly as,

$$
\begin{array}{r}
{\left[\delta w D u_{\alpha}+w \delta u^{\mu} d_{\mu} u_{\alpha}+(D w+w \partial \cdot u) \delta u_{\alpha}+\nabla_{\alpha} \delta \mathscr{P}+w D \delta u_{\alpha}+d_{\mu} \delta \Pi_{\alpha}^{\mu}-J_{\alpha}\right] \Delta^{v \alpha}=0} \\
{\left[D \delta \varepsilon+\delta w \partial \cdot u+d_{\mu}\left(w \delta u^{\mu}\right)+w \delta u^{\alpha} D u_{\alpha}-u^{\alpha} d_{\mu} \delta \Pi_{\alpha}^{\mu}+u^{\alpha} J_{\alpha}\right] u^{v}=0}
\end{array}
$$

where $w=e+\mathscr{P}$ is the enthalpy density. The perturbations of stress tensor are $\delta \Pi^{\mu \nu}$. 
To determine the source term in the jet-medium interaction, we follow a kinetic approach of the evolution of the jet parton [18]. In terms of an effective phase space distribution function $f\left(t, \vec{x}, \omega, \vec{k}_{\perp}\right)$ of an energetic jet parton, the kinetic equation is

$$
\frac{d}{d t} f\left(t, \vec{x}, \omega, \vec{k}_{\perp}\right)=\left[\hat{e} \frac{\partial}{\partial \omega}+\frac{1}{4} \hat{q} \frac{\partial}{\partial \vec{k}_{\perp}^{2}}\right] f\left(t, \vec{x}, \omega, \vec{k}_{\perp}\right),
$$

where parameters $\hat{e}$ specifies energy loss, and $\hat{q}$ is the transverse momentum broadening parameter. Given Eq. (2.6), the contribution to the source term in hydro from the jet parton energy loss can be found as

$$
-\int \frac{d^{3} \vec{k}}{\omega} k^{\mu} k^{v} \partial_{v} f=u_{j e t}^{\mu} \hat{e} \int d^{3} \vec{k} f=u_{j e t}^{\mu} \hat{e} n_{j e t}(\vec{x}, t),
$$

The above derivation is exact regarding a massless jet parton, and the jet parton velocity is $u_{\text {jet }}^{\mu}=$ $(1, \overrightarrow{1})$. The density of the jet parton $n_{\text {jet }}$ leads to a space-time dependence of the source. Especially, it characterizes the trajectory of the jet parton going through the medium. Similarly, the second term related to the momentum broadening leads to

$-\frac{\hat{q}}{4} u_{j e t}^{\mu} \int d^{3} k\left(\frac{\partial^{2} \omega}{\partial k_{\perp}^{i 2}}\right) f\left(t, \vec{x}, \omega, \vec{k}_{\perp}\right)=-\frac{\hat{q}}{4} u_{j e t}^{\mu} \int d^{3} k \frac{1}{\omega}\left(2-\frac{k_{\perp}^{2}}{\omega^{2}}\right) f\left(t, \vec{x}, \omega, \vec{k}_{\perp}\right) \sim-\frac{\hat{q}}{2} u_{j e t}^{\mu} n_{j e t} / p_{j e t}$.

The last step is estimated by considering a high energy jet particle with only colinear emission: $f\left(t, \vec{x}, \omega, \vec{k}_{\perp}\right) \sim n_{\text {jet }}(\vec{x}, t) \delta\left(k_{\|}-p_{j e t}\right) \delta^{2}\left(\vec{k}_{\perp}\right)$. We consider the relation $\hat{q}=4 \hat{e} T$ [19] as a result of fluctuation-dissipation, so that the momentum broadening contribution is suppressed by a factor of the order of $O\left(T / p_{\text {jet }}\right)$ comparing to the source term Eq. (2.7) induced by jet parton energy loss. Therefore, we neglect the induced jet-medium interaction from the transverse momentum broadening in the evolution of the jet source, so that

$$
J^{\mu}=u_{j e t}^{\mu} \hat{e} n_{j e t}(\vec{x}, t) .
$$

Throughout this work, we take into account a $T^{2}$-dependence of the jet parton energy loss rate, i.e.,

$$
\hat{e}=\kappa T^{2}
$$

with $\kappa$ a dimensionless constant. We consider two possible scenarios to determine $\kappa$. The first concerns a weakly coupled medium system, in which the dynamical properties of system can be estimated by a quasiparticle assumption, one expects $\kappa \approx s / 3 \eta$ [20]. Namely, following the dynamics of in a weakly-coupled system, the jet parton loses more energy to the medium when the medium is less dissipative. Because the effect of jet-medium interaction is determined essentially by the amount of energy deposited from the jet parton, we expect a suppressed jet-medium interaction in a more dissipative fluid. We refer to this suppression as the dynamical viscous suppression. In the second scenario regarding a strongly coupled medium, there is no obvious relation between the coefficient $\kappa$ and $\eta / s$, except a lower bound to the rate of jet energy loss, $\kappa \approx s / 3 \eta$ [20]. Once the jet energy loss rate has little dependence on the medium dissipative properties, the effect of dynamical viscous suppression is negligible. 


\subsection{Gubser flow}

The Gubser flow is an analytical solution of a conformal fluid system, with nontrivial expansions along both the radial and the longitudinal directions [21]. The analytical solution of the Gubser flow relies on symmetries: boost invariance in the longitudinal direction and rotational symmetry in the azimuth. These conditions are roughly compatible with the ultra-central nucleusnucleus collisions in the heavy-ion experiments, and are extremely useful to simplify the solution of hydrodynamic equations of motion. Especially in the coordinate system $\bar{x}^{\mu}=(\rho, \theta, \phi, \xi)$, following the coordinate transformation between $\bar{x}^{\mu}$ and the Milne space-time, $x^{\mu}=(\tau, r, \phi, \xi)^{1}$,

$$
\begin{aligned}
\sinh \rho & =-\frac{1-q^{2} \tau^{2}+q^{2} r^{2}}{2 q \tau} \\
\tan \theta & =\frac{2 q r}{1+q^{2} \tau^{2}-q^{2} r^{2}}
\end{aligned}
$$

one observes the manifest $S O(3)$ symmetry in the $(\theta, \phi)$ coordinates

$$
d s^{2}=-d \rho^{2}+d \xi^{2}+\cosh ^{2} \rho\left(d \theta^{2}+\sin ^{2} \theta d \phi^{2}\right) .
$$

The parameter $q$ in the transformation Eq. (2.11) is a dimensional parameter to be determined by the system size. The transformation of coordinates imply transformations of hydro fields. For instance,

$$
\begin{aligned}
\varepsilon & =\tau^{-4} \bar{\varepsilon} \\
u_{\tau} & =\tau\left(\frac{\partial \rho}{\partial \tau} \bar{u}_{\rho}+\frac{\partial \theta}{\partial \tau} \bar{u}_{\theta}\right) \\
u_{r} & =\tau\left(\frac{\partial \rho}{\partial r} \bar{u}_{\rho}+\frac{\partial \theta}{\partial r} \bar{u}_{\perp}\right) \\
u_{\phi} & =\tau \bar{u}_{\phi} \\
u_{\xi} & =\tau \bar{u}_{\xi} .
\end{aligned}
$$

Note in the above equations, and in the following, we use an overbar on a quantity to denote it being in the $\bar{x}^{\mu}$ coordinate system. With respect to the $S O(3)$ symmetry in $(\theta, \phi)$, and the boost invariance in $\xi$, one is allowed to find the solution of flow four velocity of the background fluid

$$
\bar{u}^{\mu}=(1,0,0,0)
$$

Given the transformations in Eqs. (2.14), the background medium flow four velocity is recovered which gives rise to the Gubser's solution.

\footnotetext{
${ }^{1}$ The proper time is $\tau=\sqrt{t^{2}-z^{2}}$, and the space-time rapidity is defined as $\xi=\frac{1}{2} \log \frac{t+z}{t-z}$.
} 
The $S O(3)$ symmetry in $(\theta, \phi)$ allows a mode expansion in spherical harmonics. Regarding the perturbations of the hydro fields, we may write

$$
\begin{aligned}
\delta \bar{T} & =\bar{T} \sum_{l m} \int \frac{d k_{\xi}}{2 \pi} t^{l m}(\rho) Y_{l m}(\theta, \phi) e^{i k_{\xi} \xi} \\
\delta \bar{u}_{i} & =\sum_{l m} \int \frac{d k_{\xi}}{2 \pi}\left[v_{s}^{l m}(\rho) \Psi_{i}^{l m}(\theta, \phi)+v_{v}^{l m}(\rho) \Phi_{i}^{l m}(\theta, \phi)\right] e^{i k_{\xi} \xi} \\
\delta \bar{u}_{\xi} & =\sum_{l m} \int \frac{d k_{\xi}}{2 \pi} v_{\xi}^{l m}(\rho) Y_{l m}(\theta, \phi) e^{i k_{\xi} \xi} .
\end{aligned}
$$

where $Y_{k m}$ is a spherical harmonic. The functions $\Psi_{i}^{l m}$ and $\Phi_{i}^{l m}$ are vector spherical harmonics specifying scalar and vector modes in the transverse flow velocity. They satisfy the condition of a vanishing divergence and a vanishing curl, respectively. Indices $l$ amd $m$ label harmonic order. The variable $k_{\xi}$ is a variable conjugate to $\xi$. Once the perturbations of hydro fields are boost invariant, as what we consider in this work, the summation of modes depends only on the mode $k_{\xi}=0$.

With the mode decomposition in terms of (vector) spherical harmonics, the equations of motion for the jet-medium interaction Eqs. (2.5) can accordingly be written mode-by-mode,

$$
\frac{\partial \overline{\mathscr{V}}_{l m}\left(\rho, k_{\xi}\right)}{\partial \rho}=-\Gamma_{l}\left(\rho, k_{\xi}\right) \overline{\mathscr{V}}_{l m}\left(\rho, k_{\xi}\right)+\overline{\mathscr{S}}_{l m}\left(\rho, k_{\xi}\right)
$$

with

$$
\overline{\mathscr{V}}_{l m}(\rho)=\left(\begin{array}{c}
t_{l m}(\rho) \\
v_{l m}^{s}(\rho) \\
v_{l m}^{\xi}(\rho) \\
v_{l m}^{v}(\rho)
\end{array}\right)
$$

$\Gamma_{l}$ is a matrix whose form was given in Ref. [21]. Note that $\Gamma_{l}$ does not depend on $m$. Source $\overline{\mathscr{S}}$ is given by the mode decomposition of the jet source $\bar{J}^{\mu}$ (the jet source expressed in the $\bar{x}^{v}$ coordinate system), which leads to

$$
\begin{aligned}
\bar{J}_{\rho} & =\sum_{l m} \int \frac{d k_{\xi}}{2 \pi} c_{\rho}^{l m} Y_{l m}(\theta, \phi) e^{i k_{\xi} \xi} \\
\bar{J}_{i} & =\sum_{l m} \int \frac{d k_{\xi}}{2 \pi}\left[c_{s}^{l m} \Psi_{i}^{l m}(\theta, \phi)+c_{v}^{l m} \Phi_{i}^{l m}(\theta, \phi)\right] e^{i k_{\xi} \xi} \\
\bar{J}_{\xi} & =\sum_{l m} \int \frac{d k_{\xi}}{2 \pi} c_{\xi}^{l m} Y_{l m}(\theta, \phi) e^{i k_{\xi} \xi} .
\end{aligned}
$$

and correspondingly,

$$
\overline{\mathscr{S}}_{l m}(\rho)=\left(\begin{array}{c}
-\frac{1}{3 \overline{\bar{w}}} c_{l m}^{\rho}(\rho) \\
-\frac{2 \bar{T} \tanh \rho}{3 \bar{w} \bar{T}^{\prime}} c_{l m}^{s}(\rho) \\
\bar{T} \overline{\bar{w}\left(\bar{T}+H_{0} \tanh \rho\right)} c_{\xi}^{l m}(\rho) \\
-\frac{2 \bar{T} \tanh \rho}{3 \bar{w} \bar{T}^{\prime}} c_{v}^{l m}(\rho)
\end{array}\right)
$$

We solve numerically Eq. (2.17) for each mode, and the resulting hydro fields are obtained after mode summations according to Eqs. (2.16). In practical simulations, we notice that the evolution 
of higher order modes receive stronger viscous suppressions, proportional to $\exp \left(-l^{2} \eta / s\right)$ [21]. This sort of suppression is purely a consequence of the fluid dynamics. We refer the suppression of mode evolution due to viscosity to as the hydrodynamical viscous suppression.

\section{Jet-medium interaction in heavy-ion collisions}

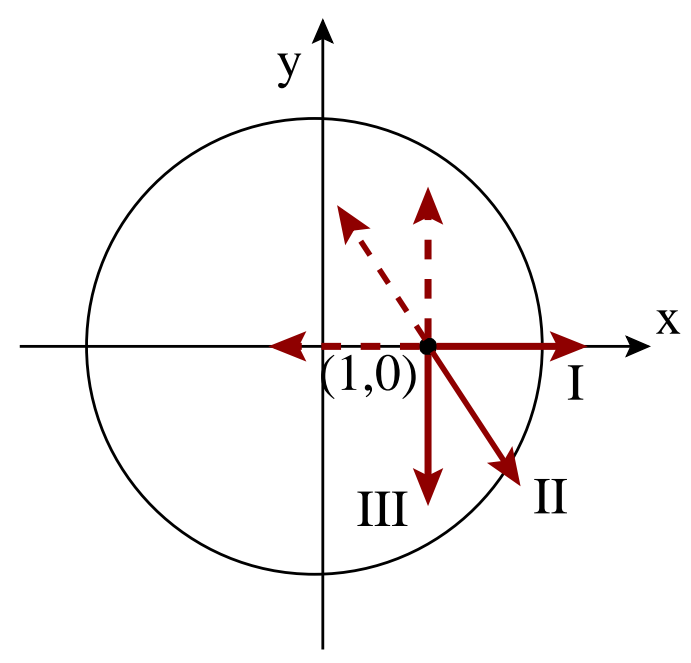

Figure 1: (Color online) Three events with di-jet considered in this work. Solid arrows indicate jet partons generating the near-side (leading) peak of the observed spectrum, while dashed arrows are those generating the away-side (sub-leading) peak.

One may adjust the entropy production of the background fluid system in the Gubser flow, according to the experimentally-measured collision events. In this work, our Gubser flow solution of the background fluid is set to approximate the ultra-central $\mathrm{Pb}+\mathrm{Pb}$ collisions at the $\mathrm{LHC} \sqrt{s_{N N}}=$ $2.76 \mathrm{TeV}[22,12]$. On top of the medium expansion, we introduce the source from the external jet parton. We consider three representative cases of the di-jet partons going through the fluid system, with their configurations illustrated in Fig. 1. In the case I, two back-to-back energic partons move along the x-axis, starting from the position $(1,0)$, at a proper time $\tau_{0}=1 \mathrm{fm} / \mathrm{c}$. For the case II and III, the pair of partons start from the same position and time, but are oriented with an angle of $\pi / 3$ and $\pi / 2$, respectively.

In the linearized hydro, we are allowed to calculate the jet-medium interaction from each parton individually in the analysis of di-jet. The resulted medium structure with respect to a di-jet can be obtained subsequently via a linear superposition. To investigate the effect of jet-medium interaction in the fluid system, we first focus on the jet-medium interaction with respect to one single jet parton. The results in Fig. 2 show the structure induced by the jet-medium interaction associated with the energetic jet parton going outwards in case I. These figures correspond to the perturbations of energy density $\delta e$ (left panel), and the energy flux along the jet parton $\delta T^{0 \|}$ (right panel), with the specific viscosity $\eta / s=1 / 4 \pi$ (upper panel) and $\eta / s=2 / 4 \pi$ (lower panel).

As one expects, the fluid response to a supersonic object results in a conical flow structure, as seen in Fig. 2. In a static medium the generated conical flow has a cone angle $\theta^{M}$ determined by the 


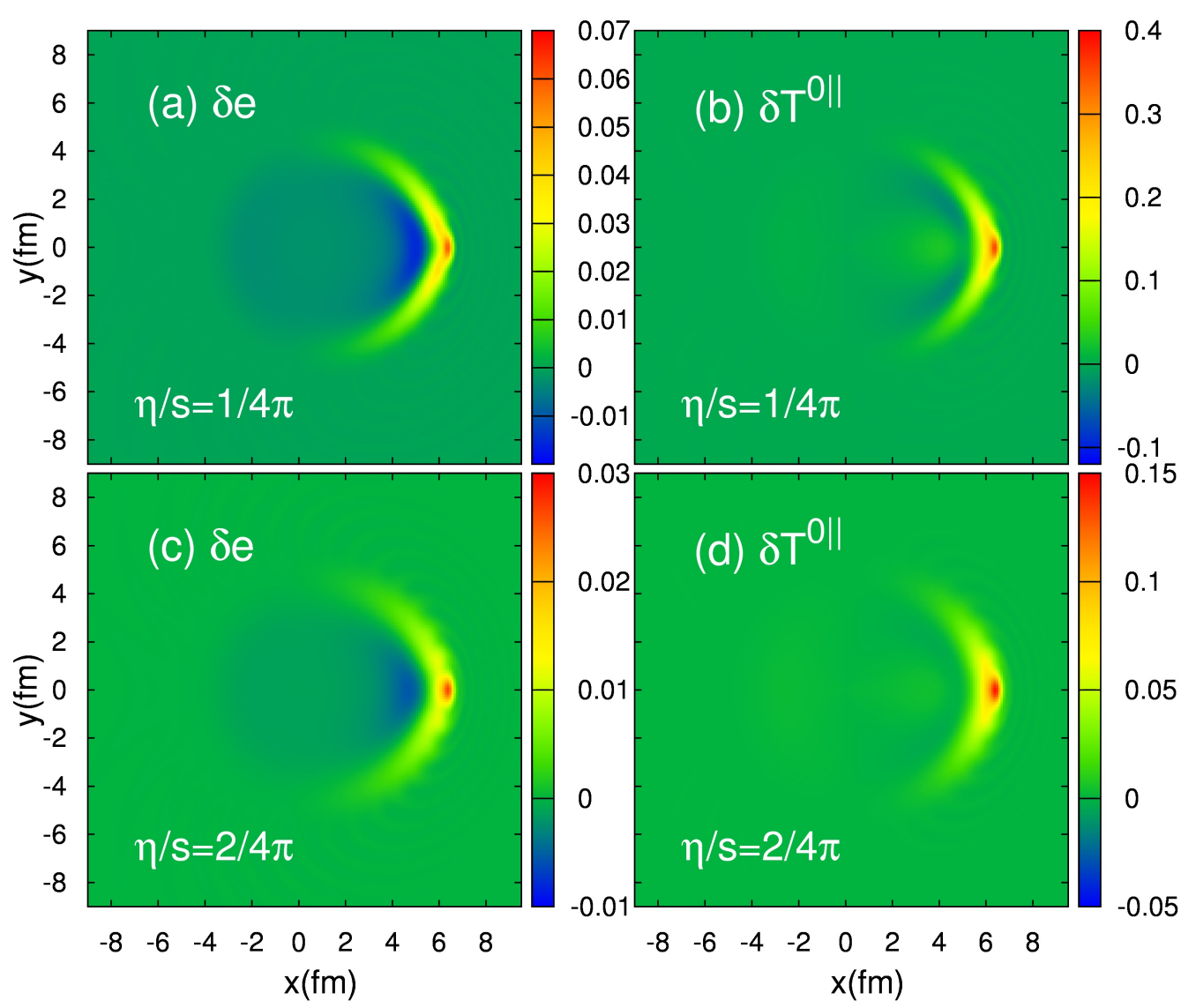

Figure 2: (Color online) Medium excitations on top of expanding viscous systems with $\eta / s=1 / 4 \pi$ (upper row) and $\eta / s=2 / 4 \pi$ (lower row) with respect to the near-side jet parton of event I, plotted in terms of energy density $\delta e$ (left panels) and energy flux $\delta T^{0 \|}$ (right panels), at $\tau=6.0 \mathrm{fm} / \mathrm{c}$. The colour coding reflects units of $\mathrm{GeV} / \mathrm{fm}^{3}$.

ratio of the parton velocity and the speed of sound: $\theta_{0}^{M}=2 \sin ^{-1}\left(c_{s} / c\right) \approx 70^{\circ}[7,6]$. The cone, and the cone angle, are both a consequence of coherent superposition of the sound wave propagation. However, with an expanding the background fluid, the propagation of sound modes is distorted. In the case shown in Fig. 2, the radial expansion tends to push out the generated cone structure, hence resulting in a larger cone angle, when comparing with $\theta_{0}^{M}$. Unlike the perturbations of energy density $\delta e$, the energy flux gets additional contributions from diffusive modes [16]. These diffusive modes lead to a diffusive wake in $\delta T^{0 \|}$ behind the shock, which however is not visible in $\delta e$.

Varying the specific viscosity from $1 / 4 \pi$ to $2 / 4 \pi$ in these calculations allows us the examine the effect of viscosity on the resulted cone structure from the jet-medium interaction. As expected, viscosity suppresses the effect of jet-medium interaction, as seen from the smearing of the cone, and a drop in total magnitudes. Regarding the dynamical viscous suppression, we expect an explicit suppression of the magnitude everywhere by a factor of 2 . On the other hand, the smearing of the cone is a consequence of the fluid response to the external parton source, and thus purely the result of hydrodynamical viscous suppression. Note that in terms of the suppression of magnitudes, 
dynamical viscous suppression is the dominant effect.

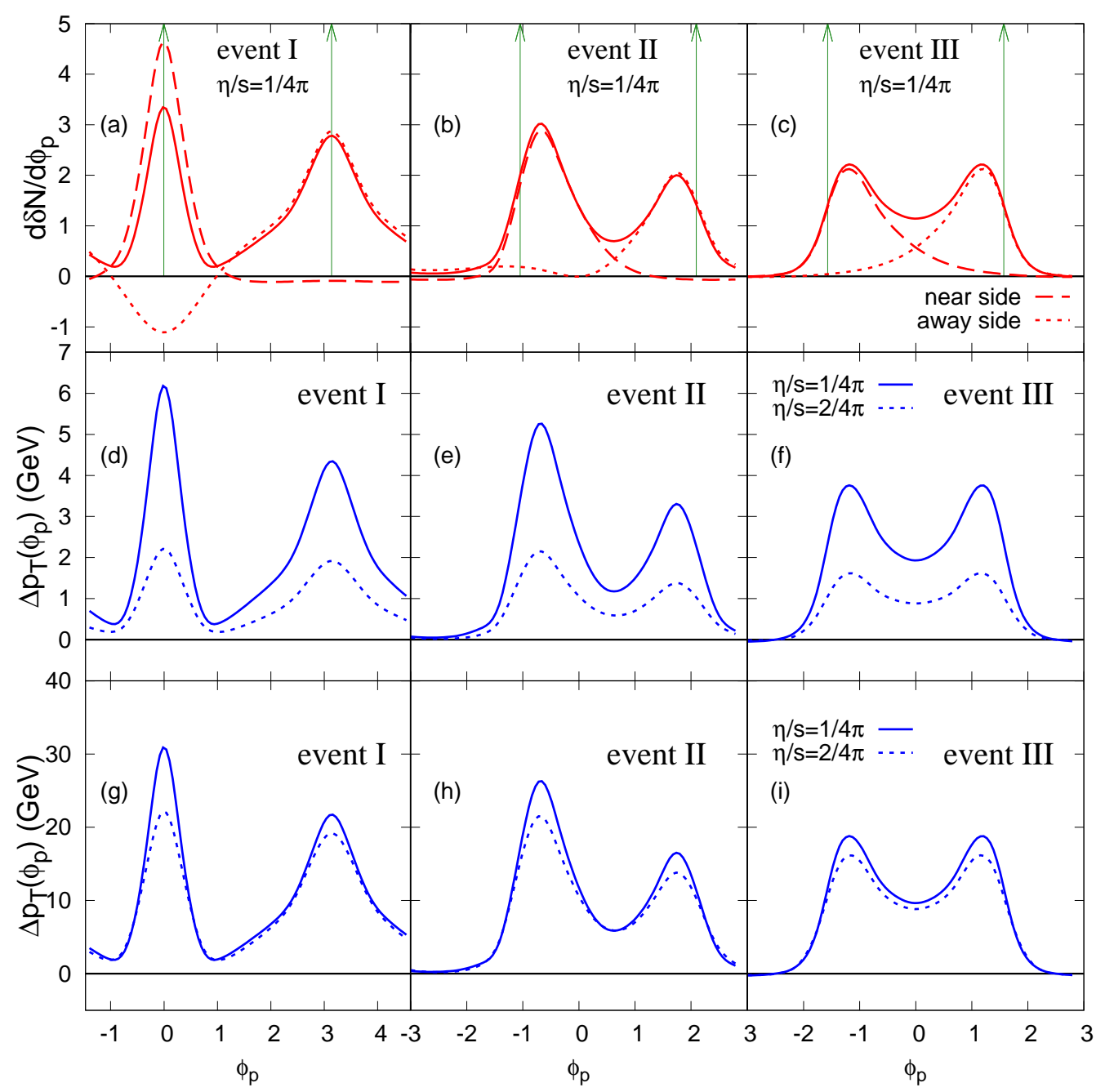

Figure 3: (Color online) Top row: pion number density generated from the medium response to jet partons as a function of azimuthal angle. The near- and away-side number density are shown separately, as well as their sum (solid line). Middle row: transverse energy of the induced pions from the medium response to a di-jet in a weakly-coupled system. Bottom row: transverse energy of the induced pions from jet-medium interaction for a strongly-coupled system. See the main text for more details. A lower cut of $p_{T} \geq 1 \mathrm{GeV}$ has been applied.

A standard Cooper-Frye freeze-out prescription can be applied to the cone structure to convert the medium response to the jet-medium interaction into the observed particle spectrum. To do so, we consider a freeze-out of the whole system - the background and the conical flow - at a proper time $\tau_{f}=6 \mathrm{fm} / \mathrm{c}$. The modification to the particle spectrum due to the jet-medium interaction is

$$
E \frac{d \delta N}{d^{3} p}=\int d \Sigma_{\mu} p^{\mu} \delta f
$$

In Eq. (3.1), the function $\delta f$ is the difference of the phase-space distribution at freeze-out, between a system with and without external jet source. The viscous correction to $\delta f$ is included as well. 
For simplicity, we only calculate the spectrum of pion, and ignore any further interactions among hadrons.

Fig. 3 displays the obtained spectrum associated with the di-jet in case I (left panel), II (middle panel) and III (right panel). Although there are two peaks observed in all these cases, associated with the pair of jet partons in the di-jet, the background medium expansion distorts differently the resulted peak structures. Basically, the width of the peak is related to the size of the conical structure as a consequence of the Cooper-Frye freeze-out prescription that converts the fluid cells on the shock structure into particles. The larger the cone, the narrower a peak one finds in the particle spectrum. For the jet parton going against the medium expansion, a wider peak is expected, as shown as the dotted lines in Fig. 3 (a) and (b). The trajectory of the back-to-back partons are identical in case III, thus one find the identical two peaks in Fig. 3 (c). The viscous suppression is revealed in Fig. 3 (d), (e) and (f) for the weakly couple system, and Fig. 3 (g), (h) and (i) for the strongly coupled system. It should be emphasized that the difference between a weekly coupled system and a strongly coupled system, in this work, only lies in the determination of the rate of energy loss. While for the weakly coupled system we take the constant $\kappa$ inversely proportional to $\eta / s$, in the strongly coupled system $\kappa$ is a constant (somehow large to be compatible with the physics of strong coupling) independent of $\eta / s$. We note that in a weakly coupled system, the viscous suppression of the spectrum comes from both the dynamical viscous suppression and the hydrodynamical viscous suppression, the viscous effect is strong. However, the dominant effect of viscous suppression is the dynamical viscous suppression. With only hydrodynamical viscous suppression, as in the strongly coupled system, the viscous effect on the generated particle spectrum is not significant. In fact, it only causes a sizeable reduction at the centers of the peaks.

\section{Summary}

In this work, we calculated the jet-medium interaction on an mode-by-mode basis. The calculation is semi-analytical since the background fluid evolution is analytically solved in the Gubser flow. Although it is a simplified analysis owing to the fact that we have taken into account a boost invariant configuration, including for the jet parton, the observed effect from viscous suppression is generic. Especially, we realize that there are two sources of viscous suppression in the obtained conical flow and the generated particle spectrum: the dynamical viscous suppression and the hydrodynamical viscous suppression. The dynamical viscous suppression accounts for the influence of viscosity on the jet parton energy loss, while the hydrodynamical viscous suppression is purely a consequence of the fluid dynamics. Although hydrodynamical viscous suppression leads to smearing the conical flow, and hence a distortion in the observed particle spectrum, the dominant viscous suppression is the dynamical viscous suppression.

\section{Acknowledgements}

This work was supported in part by the Natural Sciences and Engineering Research Council of Canada. C. G. gratefully acknowledges support from the Canada Council for the Arts through its Killam Research Fellowship program. 


\section{A. Quadratic order hydrodynamics and jet-medium interaction}

In the coupled equations of motion from the conservation of energy-momentum, Eq. (2.1), we associate the perturbations induced by the jet parton with a parameter $\lambda$. The energy-momentum tensor can be expanded in $\lambda$ as

$$
\begin{aligned}
& T_{\text {hydro }}^{\mu v}=T_{\text {hydro }}^{\mu v(0)}+\lambda T_{\text {hydro }}^{\mu v(1)}+\lambda^{2} T_{\text {hydro }}^{\mu v(2)}+O\left(\lambda^{3}\right) \\
& T_{\text {jet }}^{\mu v}=\lambda T_{\text {jet }}^{\mu v(0)}+\lambda^{2} T_{\text {jet }}^{\mu v(1)}+O\left(\lambda^{3}\right)
\end{aligned}
$$

Note that, the above expansion of the energy-momentum tensor of the jet parton starts from $O(\lambda)$, because $T_{\text {jet }}^{\mu v} \propto T^{2} n_{\text {jet }}$. Substituting the above expansions back into Eq. (2.1) and equating orders, one has

$$
\begin{aligned}
& \partial_{\mu} T_{\text {hydro }}^{\mu v(0)}=0, \\
& \partial_{\mu} T_{\text {hydro }}^{\mu v(1)}=-\partial_{\mu} T_{\text {jet }}^{\mu v(0)}=J^{v(0)}, \\
& \partial_{\mu} T_{\text {hydro }}^{\mu v(2)}=-\partial_{\mu} T_{\text {jet }}^{\mu v(1)}=J^{v(1)},
\end{aligned}
$$

Note that the form of $J^{\mu(n)}$ can be determined explicitly according to Eq. (2.9). One recognizes the first two equations at the linearized order hydrodynamics, as the coupled equations of motion, Eq. (2.3), by identifying $\delta T_{\text {hydro }}^{\mu v}=T_{\text {hydro }}^{\mu v(1)}$. By construction, the effective source term $J^{v(n)}$, depends on the perturbations of hydro fields up to $n$-th order. For instance, in the linearized hydro, $J^{v(0)}$ depends on the background hydro fields, $e^{(0)}, \mathscr{P}^{(0)}$ etc. When the series of equations are truncated at the next order, i.e., the quadratic order of hydrodynamics, one needs to solve first the linearized equations for the linearied perturbations of hydro fields, $e^{(1)}, \mathscr{P}^{(1)}$, etc., so as to determine the source term $J^{v(1)}$. Once the quadratic order hydro is solved (the third equation in Eq. (A.3)), the perturbations of hydro fields can be determined as

$$
\begin{aligned}
& \delta e=e^{(1)}+e^{(2)}, \\
& \delta \mathscr{P}=\mathscr{P}^{(1)}+\mathscr{P}^{(2)}, \\
& \delta u^{\mu}=u^{\mu(1)}+u^{\mu(2)} .
\end{aligned}
$$

\section{References}

[1] Bjorken J D Energy Loss of Energetic Partons in Quark - Gluon Plasma: Possible Extinction of High $\mathrm{p}(\mathrm{t})$ Jets in Hadron - Hadron Collisions fERMILAB-PUB-82-059-THY, FERMILAB-PUB-82-059-T

[2] Gyulassy M, Vitev I, Wang X N and Zhang B W 2003 (Preprint nucl-th/0302077)

[3] Baier R, Dokshitzer Y L, Mueller A H, Peigne S and Schiff D 1997 Nucl. Phys. B483 291-320 (Preprint hep-ph/9607355)

[4] Khachatryan V et al. (CMS) 2016 JHEP 01006 (Preprint 1509.09029 )

[5] Chatrchyan S et al. (CMS) 2011 Phys. Rev. C84 024906 (Preprint 1102 . 1957) 
[6] Casalderrey-Solana J, Shuryak E V and Teaney D 2005 J. Phys. Conf. Ser. 27 22-31 [Nucl. Phys.A774,577(2006)] (Preprint hep-ph / 0411315 )

[7] Chesler P M and Yaffe L G 2008 Phys. Rev. D78 045013 (Preprint 0712 . 0050 )

[8] Tachibana Y and Hirano T 2014 Phys. Rev. C90 021902 (Preprint 1402. 6469)

[9] Qin G Y, Majumder A, Song H and Heinz U 2009 Phys. Rev. Lett. 103152303 (Preprint $0903.2255)$

[10] Betz B, Noronha J, Torrieri G, Gyulassy M, Mishustin I and Rischke D H 2009 Phys. Rev. C79 034902 (Preprint 0812.4401 )

[11] Chaudhuri A K and Heinz U 2006 Phys. Rev. Lett. 97062301 (Preprint nucl-th/0503028)

[12] Shuryak E and Staig P 2013 Phys. Rev. C88 054903 (Preprint 1307. 2568)

[13] Chen W, Cao S, Luo T, Pang L G and Wang X N 2017 (Preprint 1704.03648 )

[14] Milhano J G, Wiedemann U A and Zapp K C 2017 (Preprint 1707. 04142)

[15] Floerchinger S and Zapp K C 2014 Eur. Phys. J. C74 3189 (Preprint 1407.1782 )

[16] Yan L, Jeon S and Gale C 2017 (Preprint 1707 . 09519)

[17] Iancu E and Wu B 2015 JHEP 10155 (Preprint 1506.07871 )

[18] Tachibana Y, Chang N B and Qin G Y 2017 (Preprint 1701.07951 )

[19] Moore G D and Teaney D 2005 Phys. Rev. C71 064904 (Preprint hep-ph / 0412346 )

[20] Majumder A, Muller B and Wang X N 2007 Phys. Rev. Lett. 99192301 (Preprint hep-ph/0703082)

[21] Gubser S S and Yarom A 2011 Nucl. Phys. B846 469-511 (Preprint 1012 . 1314)

[22] Yan L and Gronqvist H 2016 JHEP 03121 (Preprint 1511. 07198) 